\title{
CORRECTIONS
}

\section{Only the mother of all reorganisations can save the NHS}

Bob Morley is a member of the BMA's General Practitioners Committee, not the General Medical Council as stated in his competing interests (BMJ 2015;350:h1488, doi:10.1136/bmj. h1488). 